NASA/CR-2006-214294

NIA Report No. 2005-05
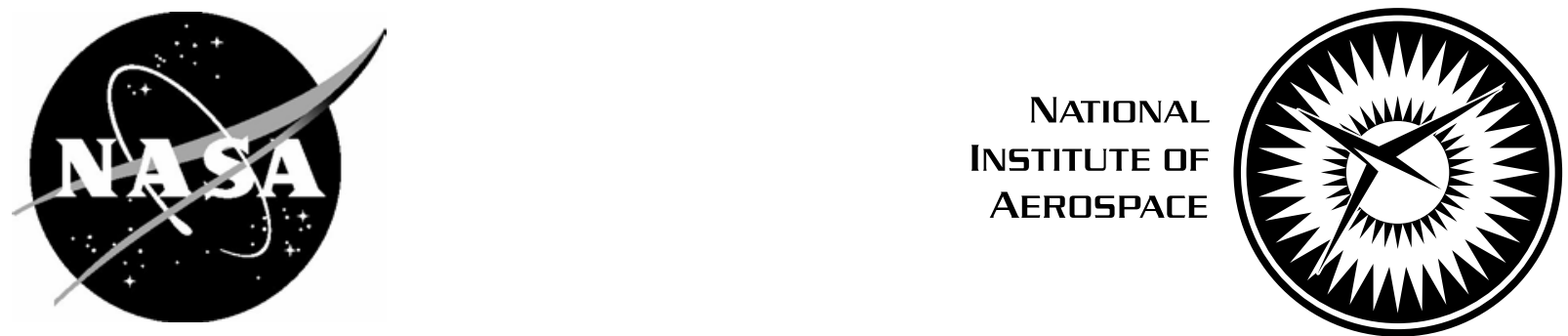

\title{
Stable Artificial Dissipation Operators for Finite Volume Schemes on Unstructured Grids
}

Magnus Svard

Stanford University, Stanford, California

Jing Gong

Uppsala University, Uppsala, Sweden

Jan Nordstrom

The Swedish Defense Research Agency, Stockholm, Sweden and

Uppsala University, Uppsala, Sweden 
Since its founding, NASA has been dedicated to the advancement of aeronautics and space science. The NASA Scientific and Technical Information (STI) Program Office plays a key part in helping NASA maintain this important role.

The NASA STI Program Office is operated by Langley Research Center, the lead center for NASA's scientific and technical information. The NASA STI Program Office provides access to the NASA STI Database, the largest collection of aeronautical and space science STI in the world. The Program Office is also NASA's institutional mechanism for disseminating the results of its research and development activities. These results are published by NASA in the NASA STI Report Series, which includes the following report types:

- TECHNICAL PUBLICATION. Reports of completed research or a major significant phase of research that present the results of NASA programs and include extensive data or theoretical analysis. Includes compilations of significant scientific and technical data and information deemed to be of continuing reference value. NASA counterpart of peerreviewed formal professional papers, but having less stringent limitations on manuscript length and extent of graphic presentations.

- TECHNICAL MEMORANDUM. Scientific and technical findings that are preliminary or of specialized interest, e.g., quick release reports, working papers, and bibliographies that contain minimal annotation. Does not contain extensive analysis.

- CONTRACTOR REPORT. Scientific and technical findings by NASA-sponsored contractors and grantees.
- CONFERENCE PUBLICATION. Collected papers from scientific and technical conferences, symposia, seminars, or other meetings sponsored or co-sponsored by NASA.

- SPECIAL PUBLICATION. Scientific, technical, or historical information from NASA programs, projects, and missions, often concerned with subjects having substantial public interest.

- TECHNICAL TRANSLATION. Englishlanguage translations of foreign scientific and technical material pertinent to NASA's mission.

Specialized services that complement the STI Program Office's diverse offerings include creating custom thesauri, building customized databases, organizing and publishing research results ... even providing videos.

For more information about the NASA STI Program Office, see the following:

- Access the NASA STI Program Home Page at http://www.sti.nasa.gov

- E-mail your question via the Internet to help@sti.nasa.gov

- Fax your question to the NASA STI Help Desk at (301) 621-0134

- Phone the NASA STI Help Desk at (301) 621-0390

- Write to:

NASA STI Help Desk

NASA Center for AeroSpace Information

7121 Standard Drive

Hanover, MD 21076-1320 
NASA/CR-2006-214294

NIA Report No. 2005-05
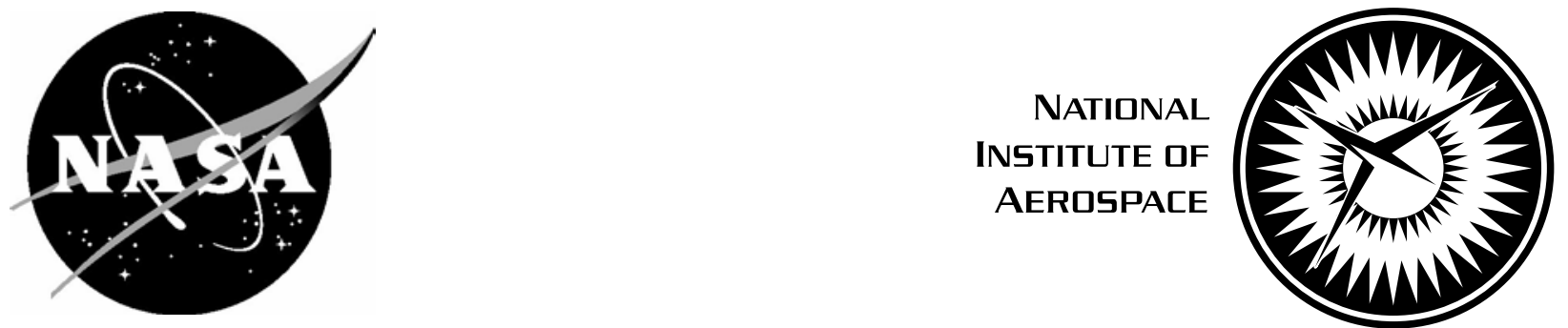

\title{
Stable Artificial Dissipation Operators for Finite Volume Schemes on Unstructured Grids
}

\author{
Magnus Svard \\ Stanford University, Stanford, California \\ Jing Gong \\ Uppsala University, Uppsala, Sweden \\ Jan Nordstrom \\ The Swedish Defense Research Agency, Stockholm, Sweden and \\ Uppsala University, Uppsala, Sweden
}

National Aeronautics and

Space Administration 
Available from:

NASA Center for AeroSpace Information (CASI)

7121 Standard Drive

Hanover, MD 21076-1320

(301) 621-0390
National Technical Information Service (NTIS) 5285 Port Royal Road Springfield, VA 22161-2171

(703) 605-6000 


\title{
Stable Artificial Dissipation Operators for Finite Volume Schemes on Unstructured Grids
}

\author{
Magnus Svärd; Jing Gong ${ }^{\dagger}$ and Jan Nordström
}

\begin{abstract}
Our objective is to derive stable first-, second- and fourth-order artificial dissipation operators for node based finite volume schemes. Of particular interest are general unstructured grids where the strength of the finite volume method is fully utilised.

A commonly used finite volume approximation of the Laplacian will be the basis in the construction of the artificial dissipation. Both a homogeneous dissipation acting in all directions with equal strength and a modification that allows different amount of dissipation in different directions are derived. Stability and accuracy of the new operators are proved and the theoretical results are supported by numerical computations.
\end{abstract}

\section{Introduction}

In computational fluid dynamics, edge based finite volume (FV) approximations are widely used (see $[1,2,3,4,5,6,7,8])$. The main advantage of those schemes is a property called grid transparency by Haselbacher et al in [1]. Grid transparancy means that the algorithm only needs information about what nodes connect to each other and thus works equally well on structured as well as unstructured grids. This property is essential for efficiency and applicability of the scheme.

${ }^{*}$ Center for Turbulence Research, Building 500, Stanford University, Stanford, CA 94305-3035, USA, e-mail: svard@stanford.edu

${ }^{\dagger}$ Department of Information Technology, Uppsala University, Uppsala, Sweden.

${ }^{\ddagger}$ Department of Computational Physics, Division of Systems Technology, The Swedish Defense Research Agency, SE-164 90 Stockholm, Sweden and Department of Information Technology, Uppsala University, Uppsala, Sweden. 
Numerical computations often require artificial dissipation to remove unphysical high-frequency oscillations. Usually second- or fourth-order artificial dissipation are used. (The use of order refers to the order of accuracy.) When shocks are present first-order dissipation need to be used. (See for example [9] and [10].) The following properties are desirable. The artificial dissipation should:

1. Reduce spurious oscillations.

2. Preserve the order of accuracy of the numerical scheme.

3. Preserve stability of the numerical scheme.

Property 1 is achieved by using undivided differences. To preserve the accuracy (Property 2) of the numerical scheme a sufficiently high-order derivative corresponding to the undivided difference need to be used. For a fourth-order accurate numerical scheme, a fourth-order undivided difference is added. One could also add a second-derivative approximation scaled with the grid size to obtain fourth-order accuracy. However, that implies that the damping of the highest frequency goes to zero as the grid is refined. With a fourth-order undivided difference the damping of the highest frequency is independent of the grid size. The treatment of Properties 1 and 2 is well-known and a variety of different dissipations have been proposed (See [9]). However, for unstructured finite volume schemes Property 3 have recieved little attention until now. We will focus on stability properties to obtain different artificial dissipation operators that satisfy all three properties even on unstructured grids.

In [6], Nordström et al considered a first derivative approximation such that convective terms can be implemented in a stable and accurate manner. The stability proofs include boundary conditions since the scheme is interpreted in a summation-by-parts framework. This work was continued in [11] where an approximation of the Laplacian was interpreted in the same framework such that schemes consisting of first derivatives and Laplacians can be proven stable. In this paper we aim to construct first-, second- and fourth-order dissipation and in order not to destroy the stability of the main scheme, the artificial dissipation has to be bounded in the same norm as the main scheme. In [6] and [11] the norm is weighted with the finite volumes that discretise the domain.

The contents of this report are divided as follows; in section 2 the general finite volume approximation is derived; in section 3 a second-order dissipation is derived; section 4 contains a derivation of a fourth-order dissipation 
operator; in section 5 the dissipation operator is modified to act with different strength in different directions. Section 6 shows numerical computations using the new dissipation operators and in section 7 conclusions are drawn.

\section{The Approximation of the Laplacian}

We aim to construct dissipation operators based on the application of the Laplacian. Therefore, we begin by deriving the standard node centred finite volume approximation of the Laplacian (see [1, 2, 3, 4, 5, 11] ). Since our main interest is to prove stability for the time dependent problem, we consider the heat equation,

$$
u_{t}=\Delta u \text {. }
$$

Integration of (1) over the domain $V_{i}$ yields,

$$
\int_{V_{i}} u_{t} d v=\int_{\partial V_{i}} \frac{\partial u}{\partial N} d s
$$

where Gauss' theorem is used. $N$ denotes the outward pointing unit normal vector such that $\frac{\partial u}{\partial N}=u_{N}=\nabla u \cdot N$. Further, let $V_{i}$ be an n-sided polygon with sides $d s_{i n}$.

Given any grid, let $r_{i}$ denote a grid point. With a slight abuse of notation we let $V_{i}$ be defined as the measure of the volume inside the so called dual grid around $r_{i}$. The dual grid is in turn defined as the straight lines drawn between the centres of mass of the cells with $r_{i}$ as a vertex and the midpoints of the edges from $r_{i}$, see Figure 1 .

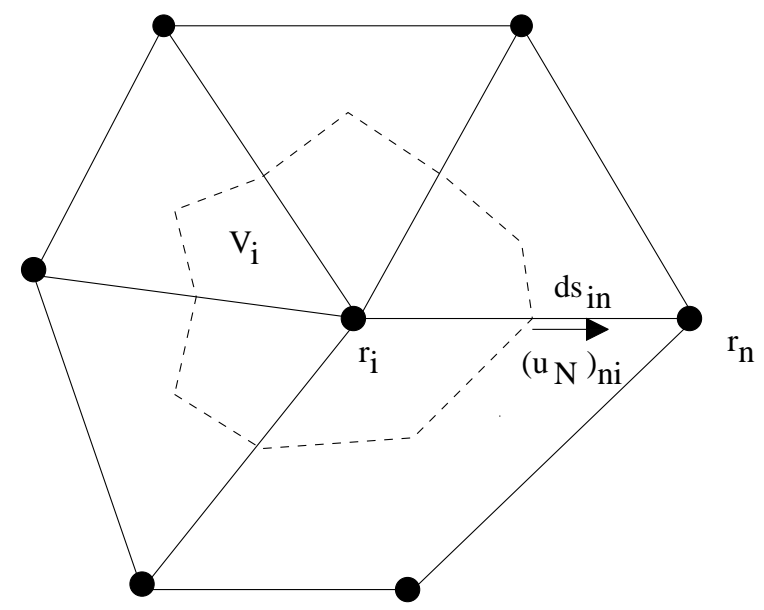

Figure 1: A generic 2D grid. Solid lines are the grid lines and dashed lines correspond to the dual grid. 
Further, $d s_{i n}$ is defined as the sum of the length of the "centre of massmidpoint-centre of mass" lines passing over one edge (see Figure 1). Let $r_{n i}=\left|r_{i}-r_{n}\right|$. Finally, let $N_{i}$ denote the set of indices of points being neighbours to $r_{i}$.

For an interior point, $r_{i}$, an approximation of (2) is,

$$
V_{i} \cdot\left(v_{t}\right)_{i}=\sum_{n \in N_{i}} \frac{v_{n}-v_{i}}{r_{n i}} d s_{i n} .
$$

and for a boundary point $b$,

$$
V_{b} \cdot\left(v_{t}\right)_{b}=\sum_{n \in N_{b}} \frac{v_{n}-v_{b}}{r_{n b}} d s_{b n}+\left(v_{N}\right)_{b} d s_{b b},
$$

where $d s_{b b}$ is the length between two midpoints of edges at the boundary. The scheme (3) and (4) can be summarised in matrix form as,

$$
P v_{t}=Q_{\Delta} v=(A+D S) v
$$

where $P$ is a diagonal matrix with $V_{i}$ on the diagonal. $D S v$ holds the terms $\left(v_{N}\right)_{b} d s_{b b}$. $A v$ represents the remaining terms (essentially the scheme for the interior points). $v_{t}$ and $v$ are vectors with components $\left(v_{t}\right)_{i}$ and $v_{i}$ respectively. In [11], $A$ is proven to be a symmetric negative-definite matrix.

\section{Second-Order Artificial Dissipation}

A second-order dissipation operator is obtained by,

$$
u_{t}=\epsilon^{2} \Delta u, \quad 0 \leq x \leq 1
$$

where $\epsilon$ is a small positive number (compare with the scaling $h^{2}$ in the discrete artificial dissipation). If we apply the energy method to (6) we obtain,

$$
\int_{0}^{1} u u_{t} d x=\left.\epsilon^{2} u u_{x}\right|_{0} ^{1}-\epsilon^{2} \int_{0}^{1} u_{x}^{2} d x .
$$

We see that $\epsilon u=0$ or $\epsilon u_{x}=0$ will result in a well posed problem. (If $\epsilon \rightarrow 0$ the boundary conditions vanish. This is the analogue of numerical boundary conditions.) In the discrete setting we have,

$$
v_{t}=h^{2} P^{-1} Q_{\Delta} v
$$


To analyse the discretisation of (6) we introduce the norm $\|v\|^{2}=v^{T} P v$. Apply the energy method,

$$
\|v\|_{t}^{2}=v^{T} P v_{t}+v_{t}^{T} P v=h^{2} 2 v^{T}(A+D S) v=h^{2}\left(2 v^{T} A v+2 v^{T}(D S v)\right) .
$$

Since $A$ is negative definite the discretisation is stable if $v=\mathbf{0}$ or $D S v=\mathbf{0}$ at the boundary. $\mathbf{0}$ denotes a vector with all entries zero. With the SAT technique (Simultaneous Approximation Term, see $[12,13,14]$ ) we impose $D S v=0$ weakly by adding,

$$
h^{2} P^{-1}(D S v-\mathbf{0})
$$

to the right-hand side of (8). Finally, we have the artificial dissipation,

$$
v_{t}=h^{2} P^{-1} A v .
$$

It is easy to determine the following sizes from (4). dim denotes the number of space dimensions.

$$
\begin{aligned}
& P^{-1} Q_{\Delta} v \sim \mathcal{O}(1), \sim \mathcal{O}\left(h^{d i m-1}\right), \\
& D S v \sim d s_{b b} \sim \mathcal{O}\left(1 / V_{i}\right), \\
& P^{-1} \sim \mathcal{V} \\
& \frac{V_{i}}{d s_{i i}} \sim \mathcal{O}(h) \\
& A v \sim \mathcal{O}\left(h^{d i m-1}\right) .
\end{aligned}
$$

This leads to second-order interior accuracy and first-order boundary accuracy, which result in globally second-order accuracy.

Remark A scalar equation has been considered when deriving the artificial dissipation. That equation represents the treatment of a single variable in a system of partial differential equations such as the Euler or Navier-Stokes equations. The numerical boundary conditions discussed above, only describe how to close the scheme. They do not affect the physical boundary conditions and need not be changed if another equation is considered. Note that no boundary conditions are imposed in Equation (11) and that no boundary conditions should be imposed since it is an approximation of $u_{t}=0$.

\subsection{Some Remarks on First Order Dissipation}

It is well-kown that a shock capturing dissipation needs to be first order which can be obtained by dividing the second-order dissipation by the grid size. We propose one such scaling in Section 6 on general unstructured grids. 
Another issue is the scaling of the dissipation for linear problems. To address this question we consider two different kinds of grid independence. First, if the entire problem is rescaled such that the grid size is doubled but with the same number of points (that is a twice as big grid) and the equations are rescaled accordingly so that the exact solution at each grid point is the same on the small and big grid, then that should be the case for the numerical solution as well. We illustrate this with an example. Consider, the periodic problem $u_{t}+a u_{x}=0$ with initial data $u(x, 0)=f(x)$ on $0 \leq x<1$. Then the solution is $u(x, t)=f(\xi)$ where $\xi=x-a t+a n$ and $n$ is an integer chosen such that $\xi \in[0,1]$.

Next, consider the periodic problem $v_{t}+2 a v_{y}=0$ with $v(y, 0)=f(y / 2)=$ $f(x)$ on $0 \leq y<2$. The solution is $v(y, t)=f(\eta / 2)$ where $\eta=y-2 a t+2 a n$. $n$ is an integer chosen such that $\eta \in[0,2]$.

The two examples have identical solutions at the points located at the same ratio of the total interval. For example $u(1 / 8, t)=v(1 / 4, t)$. This is easily understood since the two problems connects through the mapping $y=2 x$ such that $u_{x}=u_{y} y_{x}=2 u_{y}$.

Next, we turn to the discrete problems on the same domains. Let $u_{t}+$ $a D_{x} u=A_{x} u$ where $D_{x}$ is the discrete $x$-derivative on $x \in[0,1]$ with grid spacing $h$ and $A_{x}$ is an artificial dissipation operator. Further, $u\left(x_{i}, 0\right)=$ $f\left(x_{i}\right)$. Let $v_{t}+2 a D_{y} v=A_{y} v$ be the same discretisation with grid spacing $2 h$ on $y \in[0,2]$ and $u\left(y_{i}, 0\right)=f\left(x_{i}\right)$. Note that we have the same number of grid points in the two problems. The two problems are indentical under the transformation $y=2 x$ and so are the discrete solutions. Hence, at grid point $i$ we have, $u\left(x_{i}, t\right)=v\left(y_{i}, t\right)$. Note that we have not assumed anything regarding the size of the dissipation so this similarity applies to both firstand second-order dissipation.

The second property of importance is the scaling of the dissipation for a given linear problem and for different grid sizes.

We consider the periodic problem $u_{t}+a u_{x}=0$ on $0 \leq x<1$ described previously in this section. It is discretised by $v_{t}+a D_{x} v=A_{x} v$ where $D_{x} v$ is the standard second-order (non-dissipative) approximation. $A_{x}=h^{j} \widetilde{A}_{x}$ where $\tilde{A}_{x} v$ is the undivided second-derivative approximation times a constant c. $j=1,2,3 \ldots$ yields a $j$ th-order dissipation. Written in component form the $N+1$-point discretisation is,

$$
\left(v_{n}\right)_{t}+a \frac{v_{n+1}-v_{n-1}}{2 h}=h^{j} c \frac{v_{n+1}-2 v_{n}+v_{n-1}}{h^{2}}, n=1 . . N, v_{0}=v_{N} .
$$


Expand the solution in a Fourier series with $h=1 / N$,

$$
\begin{array}{r}
v(t)_{n}=\sum_{m=0}^{N-1} \hat{v}(t)_{m} e^{i m \omega_{n}}, \omega_{n}=2 \pi x_{n} \in[0,2 \pi(N-1) / N], \\
\hat{v}(t)_{m}=\sum_{n=0}^{N-1} v\left(x_{n}\right) e^{-i m \omega_{n}} h .
\end{array}
$$

Insert the Fourier expansion into the error equation,

$$
\sum_{m=0}^{N-1}\left(\left(\hat{v}(t)_{m}\right)_{t} e^{i m \omega_{n}}-\hat{v}(t)_{m} e^{i m \omega_{n}}\left(-\frac{a}{h} i \sin (m 2 \pi h)+2 c h^{j-2}(\cos (m 2 \pi h)-1)\right)\right)=0 .
$$

Note that with this choice of transform $m=0$ corresponds to a constant and $m=N-1$ is the least oscillating mode while $m=N / 2$ is the most oscillating (usually called the $\pi$-mode since with $m=N / 2$ we have $m 2 \pi h=\pi$ ).

The purpose of the dissipation is to damp the unresolved modes while leaving the resolved modes undisturbed. Hence a low frequency mode should have less and less damping as the grid is refined and the highest mode should experience the same damping. Therefore, we study the behaviour of the mode $m=N / 2$. The equation for that mode is,

$$
\begin{array}{r}
\left(\hat{v}(t)_{N / 2}\right)_{t}=\hat{v}(t)_{N / 2}\left(-\frac{a}{h} i \sin (2 \pi h N / 2)+2 c h^{j-2}(\cos (2 \pi h N / 2)-1)=\right. \\
-\hat{v}(t)_{N / 2} 4 c h^{j-2} .
\end{array}
$$

Now, it is easily seen that the only $h$ independent solution is achieved with $j=2$. If $j=1$ the highest frequency will have increased damping as $h \rightarrow 0$ and if $j>2$ the damping will diminish with smaller $h$.

These conclusions hold for any constant ratio $N / x$ where $x$ is a valid choice that gives an existing mode. Hence the choice $j=2$ imposes the same damping, independent of $h$, on the portion of the modes that are unresolved. However, if a fixed mode $N=N_{\text {fix }}$ is considered the damping on that mode will decrease with $h$ since it will be more and more resolved.

The general conclusion for a $p$ th-order scheme is that the undivided difference corresponding to the $p$ th derivative gives the grid independent dissipation. 


\section{Fourth-Order Artificial Dissipation}

The most obvious fourth-order artificial dissipation using the Laplacian would be $\Delta(\Delta u)$ or in the semi-discrete case,

$$
v_{t}=-h^{4} P^{-1} Q_{\Delta} P^{-1} Q_{\Delta} v .
$$

In order to prove stability, equation (15) has to supplied with numerical boundary conditions. To investigate what numerical boundary conditions to use we examine the one-dimensional continuous counterpart of equation (15).

$$
u_{t}=-h^{4} u_{x x x x}, \quad 0 \leq x \leq 1
$$

Apply the energy method to equation (16).

$$
\int_{0}^{1} u u_{t} d x=-h^{4} \int_{0}^{1} u u_{x x x x} d x=-\left.h^{4} u u_{x x x}\right|_{0} ^{1}+\left.h^{4} u_{x} u_{x x}\right|_{0} ^{1}-h^{4} \int_{0}^{1} u_{x x}^{2} d x(
$$

In (17) it is seen that the boundary conditions $h^{4} u_{x x x}=0$ and $h^{4} u_{x}=0$ will result in a well posed problem.

Next turn to the discrete equation (15) and apply the energy method to prove stability,

$$
\begin{array}{r}
\|v\|_{t}^{2}=v^{T} P v_{t}+v_{t}^{T} P v=-h^{4}\left(v^{T}(A+D S) P^{-1}(A+D S) v+\right. \\
\left.v^{T}(A+D S)^{T} P^{-1}(A+D S)^{T} v\right)= \\
-h^{4}\left(2 v^{T} A P^{-1} A v+2 v^{T} A P^{-1} D S v+2 v^{T} D S P^{-1}(A+D S) v\right) .
\end{array}
$$

If equation (18) is compared with (17) we note that the boundary terms correspond to each other. In the discrete case we would choose,

$$
\begin{aligned}
h^{4} D S P^{-1}(A+D S) v & =\mathbf{0}, \\
h^{4} D S v & =\mathbf{0} .
\end{aligned}
$$

Equation (19) is precisely a discretisation of $h^{4} u_{x x x}=0$ and (20) is the discretisation of $h^{4} u_{x}=0$. To impose these numerical boundary conditions in a stable manner we again use the SAT technique (see $[12,13,14])$ and add the following terms to the right hand side of (15),

$$
h^{4} P^{-1} D S P^{-1}(A+D S)(v-\mathbf{0})+h^{4} P^{-1} A P^{-1} D S(v-\mathbf{0}),
$$

and obtain the artificial dissipation,

$$
v_{t}=-h^{4} P^{-1} A P^{-1} A v .
$$


To determine the size of (22) we consider the penalty terms (21). The sizes in (12) apply here also, which implies that the first penalty term in (21) is $\mathcal{O}\left(h^{3}\right)$, the second is $\mathcal{O}\left(h^{2}\right)$ and equation (15) is $\mathcal{O}\left(h^{4}\right)$. Since the action of the penalty terms is restricted to the vicinity of the boundary the global order of accuracy of $(22)$ is $\mathcal{O}\left(h^{3}\right)$.

In fact, it is immediately realised that the artificial dissipation (22) might be a good choice because it is negative definite in the $P$-norm. However, the above derivation gives a more thorough understanding of the action of (22) and is also required to understand what order of accuracy that is obtained.

The choice of numerical boundary conditions can also be understood directly from the discretisation. The action of the penalty terms is to cancel the $D S$ part of the second derivative discretisation. Thus, when this artificial dissipation is used the Laplacian algorithm is run twice on the entire domain. The first time $\left(v_{N}\right)_{b}=0$ in (4) and the second time $v_{n}$ in (3) and (4) represents $(\Delta v)_{n}$, i.e. we have $\left((\Delta v)_{N}\right)_{b}=0$. This precisely corresponds to the boundary conditions $u_{x}=0$ and $u_{x x x}=0$.

Remark In [11] it was shown that on grids different from equilateral polygons the approximation $P^{-1} Q_{\Delta} v=$ const $\cdot \Delta v+\mathcal{O}(1)$. Although, $P^{-1} Q_{\Delta}$ does not approximate the Laplacian on general grids it is always dissipative since $A$ is negative semidefinite and has the correct size. Moreover, the dissipation is always a blend of derivatives, including the Laplacian. Hence, it has the same effect as the Laplacian and the additional dissipation will not affect a constant.

Remark The fourth-order JST operator (see [9]) is built from a first-derivative approximation $D_{1}$, a third-derivative approximation $D_{3}$ and a scaling $\lambda\left(x_{i}\right)$ to become $\left(D_{1} \lambda D_{3}\right) u$. With this construction it is not possible to derive an energy estimate proving stability.

\section{Direction Dependent Artificial Dissipation}

In the previous section the artificial dissipation did not take directions into account. Sometimes, it is desirable to scale the artificial dissipation differently in different space directions. In order to include that possibility, we will modify the approximation of the Laplacian.

Following the analysis in [11] we consider,

$$
P v_{t}=A v
$$


or at a specific point $r_{i}$,

$$
V_{i}\left(v_{t}\right)_{i}=\sum_{n \in N_{i}} \frac{v_{n}-v_{i}}{r_{n i}} d s_{i n}=\sum_{n \in N_{i}} \frac{d s_{i n}}{r_{n i}} v_{n}-v_{i} \sum_{n \in N_{i}} \frac{d s_{i n}}{r_{n i}}=\sum_{n \in N_{i}} a_{i n} v_{n}+a_{i i} v_{i}
$$

where $N_{i}$ is the set of indices of neighbours to a point $r_{i}$, implying that,

$$
\begin{aligned}
a_{i i} & =-\sum_{n \in N_{i}} \frac{d s_{i n}}{r_{n i}}, \\
a_{i n} & =\frac{d s_{i n}}{r_{n i}}, \quad \text { if } n \in N_{i} .
\end{aligned}
$$

Define $a_{i j}=0$ whenever $j \notin N_{i}$. Further, $a_{i j}$ is the $(i, j)$ component of $A$.

As is shown in [11], $A$ has the following properties.

$$
\begin{array}{r}
\sum_{i \neq j} a_{i j}=\sum_{n \in N_{i}} a_{i n}=-a_{i i} \\
a_{i j}=a_{j i} \\
a_{i i}<0 .
\end{array}
$$

These properties are equivalent to $A$ being symmetric and negative semidefinite, which are necessary and sufficient for stability. That is, we may modify $A$ such that the properties (27)-(29) are not violated and still obtain a stable approximation. In the case of artificial dissipation it will also be accurate (have the correct size) due to the scaling.

Let $\hat{e}$ denote a unit vector in space in which direction the artificial dissipation should act and $\hat{n}_{i n}$ the unit vector pointing at the direction $r_{i}-r_{n}$. We propose the following modification.

$$
V_{i}\left(v_{t}\right)_{i}=\sum_{n \in N_{i}} \frac{v_{n}-v_{i}}{r_{n i}} d s_{i n}\left|\hat{n}_{i n} \cdot \hat{e}\right|
$$

In this case,

$$
a_{i n}=\frac{d s_{i n}}{r_{n i}}\left|\hat{n}_{i n} \cdot \hat{e}\right|
$$

such that property (28) is fulfilled by definition. Also, it is easily seen that property (27) is satisfied (c.f [11]). Then (29) will also be fulfilled since $a_{i n} \geq$ 0 . The properties (27)-(29) are all satisfied, implying that this modification of the Laplacian is stable. 
Equation (30) is just one example of a Laplacian based artificial dissipation. A generalisation would be,

$$
V_{i}\left(v_{t}\right)_{i}=\sum_{n \in N_{i}} \frac{v_{n}-v_{i}}{r_{n i}} d s_{i n} c_{n i} .
$$

Stability will not be destroyed if $c_{j i}=c_{i j} \geq 0$ (c.f the above derivation and [11]). Thus, for example, it is possible to change the direction that the dissipation is acting at different parts of the grid without formally destroying stability.

Remark With this observation in mind we would be able to choose $c_{i j}=$ $\left|\hat{n}_{i j} \cdot \hat{e}_{x}+c\right|$, where $c$ is some constant, in the Cartesian case such that a nonzero dissipation is always obtained in the direction normal to $x$.

To obtain a fourth-order dissipation we would apply (31) twice and according to (22) multiply it by $h^{4}$ where $h$ is some grid size. The remaining issue is the choice of $h$. With a general unstructured grid the cell sizes may vary considerably in the domain and a better scaling should be used. However, $r_{i n}$ is the size of an edge locally and we may use that when deriving the dissipation.

$$
\begin{aligned}
\left(v_{t}\right)_{i}=h^{2} \frac{1}{V_{i}} \sum_{n \in N_{i}} \frac{v_{n}-v_{i}}{r_{n i}} d s_{i n} c_{n i} \sim & \frac{1}{V_{i}} \sum_{n \in N_{i}} r_{n i}^{2} \frac{v_{n}-v_{i}}{r_{n i}} d s_{i n} c_{n i}= \\
& \frac{1}{V_{i}} \sum_{n \in N_{i}}\left(v_{n}-v_{i}\right) r_{n i} d s_{i n} c_{n i}
\end{aligned}
$$

Again, conditions (27)-(29) are fulfilled since $r_{i n}=r_{n i} \geq 0$.

The equations (22) and (32) constitute the final artificial dissipation. (Both are similar to the artificial dissipations used in $[2,15,16]$ ). That is, at each time step (32) is used once and yields an approximation of the Laplacian with homogeneous boundary conditions, $(\widetilde{\Delta u})_{i}$, at each grid point $i$ in the domain. Equation (32) is then applied again on the grid function $(\widetilde{\Delta u})_{i}$ instead of $u_{i}$ such that the fourth order dissipation is obtained. Note that with this approach, the edge based data structure is not destroyed, maintaining an efficient implementation of the finite volume scheme.

Let us analyse the action of the proposed artificial dissipation, equation (32), on an equidistant Cartesian grid where the desired direction is $\hat{e}_{x}$. In this case we can order the solution in a matrix $\left\{v_{i j}\right\}$ where the first index 
denotes space variation in the $\mathrm{x}$-direction and the second index in the $\mathrm{y}$ direction. Let $h$ denote the grid spacing. In this case $r_{i j}=d s_{i j}=h$ and $V_{i}=h^{2}$. We have for a point $p$,

$$
\begin{array}{r}
\left(v_{t}\right)_{p p}=\frac{1}{h^{2}}\left(\left(v_{p+1, p}-v_{p, p}+v_{p-1, p}-v_{p, p}\right) h^{2}\left|\hat{e}_{x} \cdot \hat{e}_{x}\right|+\right. \\
\left.\left(v_{p, p+1}-v_{p, p}+v_{p, p-1}-v_{p, p}\right) h^{2}\left|\hat{e}_{y} \cdot \hat{e}_{x}\right|\right),
\end{array}
$$

or,

$$
\left(v_{t}\right)_{p p}=v_{p+1, p}-2 v_{p, p}+v_{p-1, p},
$$

i.e. the standard second order undivided finite difference approximation of the second derivative in the x-direction. The Laplacian used twice will of course result in the standard fourth-order undivided finite difference approximation.

\subsection{Concluding Remarks on the Fourth Order Dissi- pation}

Consider,

$$
v_{t}=-h^{4} P^{-1} A \Lambda P^{-1} A v,
$$

where $\Lambda$ is a diagonal positive definite matrix. The energy method leads to,

$$
\|v\|_{T}^{2}=-2 v^{T} A \Lambda P^{-1} A v=-h^{4} 2 v^{T} A \Lambda^{1 / 2} P^{-1} \Lambda^{1 / 2} A v,
$$

where the right-hand side is a negative-definite quadratic form. Introduce $\tilde{A}=A \Lambda^{1 / 2}$. Equation (33) becomes,

$$
v_{t}=-h^{4} P^{-1} \tilde{A}^{T} P^{-1} \tilde{A} v,
$$

$\tilde{A}=A \Lambda^{1 / 2}$ can be interpreted as $c_{i j} \neq c_{j i}$ in (31). To be more specific, $c_{n i}=c_{i i}$ for all $n \in N_{i}$ and $c_{i i}>0$ can be chosen arbitrarily. This form of dissipation does not take directions into account but scales the dissipation only with respect to position in space.

\section{Numerical Computations}

\subsection{Linear examples}

We will consider the two-dimensional advection equation.

$$
\begin{aligned}
u_{t}+a u_{x}+b u_{y} & =0, \quad(-1 \leq x \leq 0,0 \leq y \leq 1)=\Omega \\
L u & =g(x, y) \quad(x, y) \in \partial \Omega \\
u(x, y, 0) & =f(x, y)
\end{aligned}
$$


where,

$$
L=\left\{\begin{array}{ll}
1, & (a, b) \cdot \hat{n}<0 \\
0, & (a, b) \cdot \hat{n} \geq 0
\end{array},\right.
$$

and $\hat{n}$ is the unit outward pointing normal. We will use the first derivative finite volume operators defined in [6] denoted $P^{-1} Q_{x}$ and $P^{-1} Q_{y}$ in the $x$ and $y$-direction respectively. (Those are the standard scheme obtained with Green's theorem.) In [6] equations such as (36), were proven stable with a weak implementation of the boundary conditions and we refer to that article for details. The second-order artificial dissipation operator is defined in (32), and repeated here for convenience,

$$
\left(D_{2} v\right)_{i}=\frac{1}{V_{i}} \sum_{n \in N_{i}}\left(v_{n}-v_{i}\right) r_{n i} d s_{i n} c_{n i} .
$$

In the computations we use $c_{n i}=1$. To obtain a first-order dissipation we divide by $r_{n i}$,

$$
\left(D_{1} v\right)_{i}=\frac{1}{V_{i}} \sum_{n \in N_{i}}\left(v_{n}-v_{i}\right) d s_{i n} .
$$

Finally we define $D_{4} v=D_{2} D_{2} v$. A semi-discretisation of (36) is,

$$
v_{t}+a P^{-1} Q_{x} v+b P^{-1} Q_{y} v=\epsilon_{1} D_{1} v+\epsilon_{4} D_{4} u+B C,
$$

where $B C$ are penalty terms imposing the boundary conditions. Two test cases, computed on an unstructured triangular grid with 4357 nodes, are considered:

1. $\epsilon_{1}=0, \epsilon_{4}=0$ or 1 , random numbers as initial data, see Figure 2 .

2. $\epsilon_{1}=0, \epsilon_{4}=0$ or 5 , sine function with random perturbation as initial data, see Figure 2.

The results from Test 1 are displayed in Figure 3. Clearly, the dissipation damps the solution. Further, the non-dissipative computation converge very slowly to the steady state solution $u=0$. Test case 2, Figure 4, also signifies the damping performed by the dissipation operator without altering the underlying solution. 


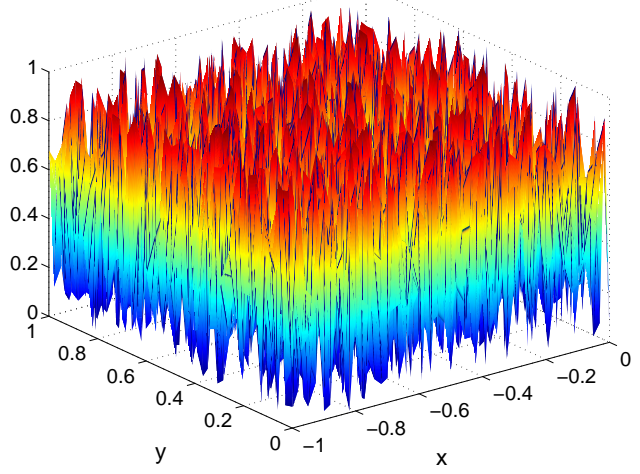

(a) Random initial data.

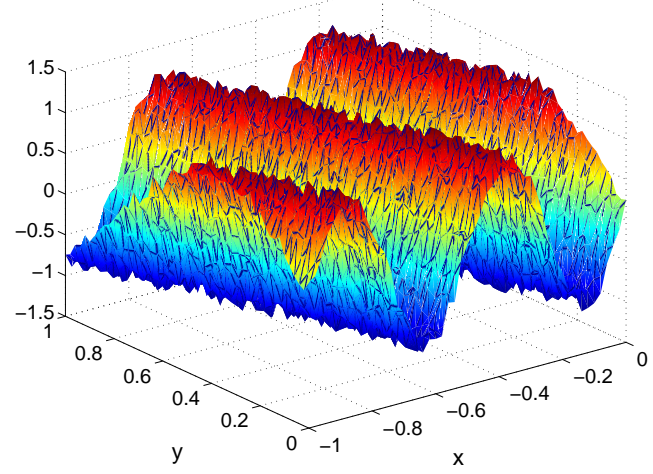

(b) Since function with random perturbation.

Figure 2: Two different initial data.

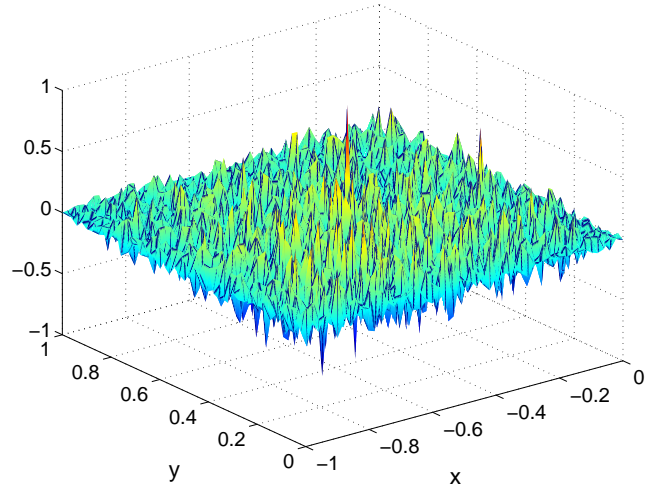

(a) Without dissipation.

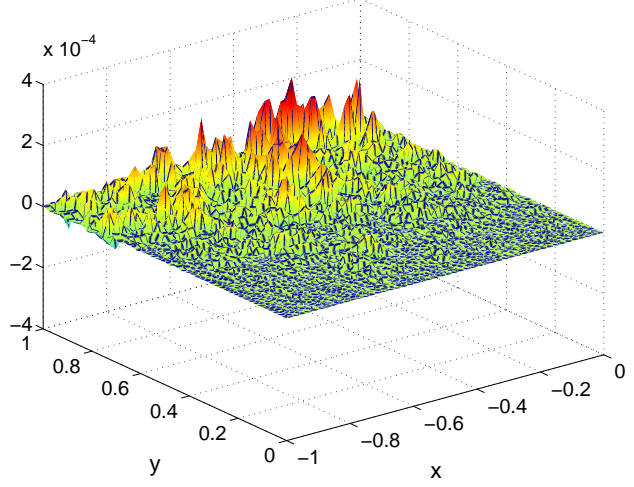

(b) With fourth-order dissipation.

Figure 3: Solution at $t=1$ with random initial data. 


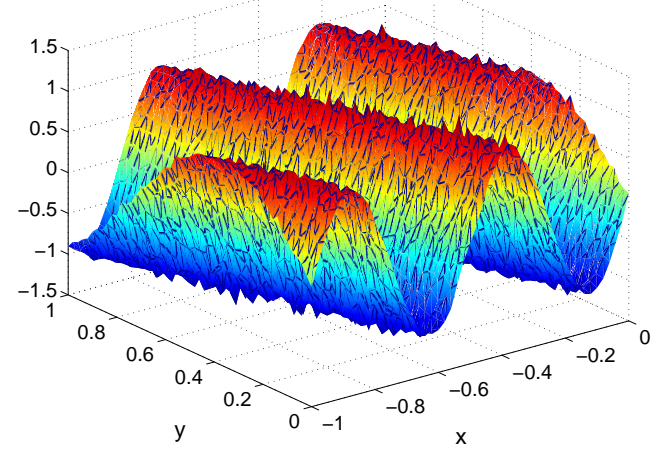

(a) Without dissipation.

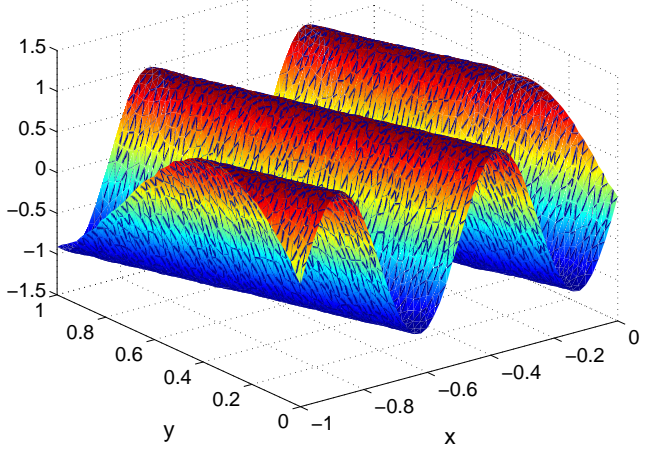

(b) With fourth-order dissipation.

Figure 4: Solution at $\mathrm{t}=0.5$ with perturbed sine function as initial data.

\subsection{Nonlinear examples}

Consider Burgers' equation,

$$
\begin{aligned}
u_{t}+\left(\frac{u^{2}}{2}\right)_{x} & =0, \quad(-1 \leq x \leq 0,0 \leq y \leq 1)=\Omega \\
u(-1, y, t) & =u_{L}, \quad u(0, y, t)=u_{R} \\
u(x, y, 0) & =f_{1,2}(x, y)
\end{aligned}
$$

where we use either,

$$
f_{1}(x, y)= \begin{cases}u_{L}=1, & -1 \leq x \leq-0.9 \\ -15 x-12.5, & -0.9 \leq x \leq-0.8 \\ u_{R}=-0.5, & -0.8 \leq x \leq-1\end{cases}
$$

or

$$
f_{2}(x, y)= \begin{cases}u_{L}=3, & -1 \leq x \leq-0.8 \\ -10 x-5, & -0.8 \leq x \leq-0.6 \\ u_{R}=1, & -0.6 \leq x \leq-1\end{cases}
$$

as initial data. We test 3 different cases where at most one of $\epsilon_{1}$ and $\epsilon_{4}$ is nonzero:

1. Initial data $f_{1}$, unstructured fine mesh (11139 nodes). $\epsilon_{1}=1, \epsilon_{4}=0$ or $\epsilon_{1}=0, \epsilon_{4}=5$. 


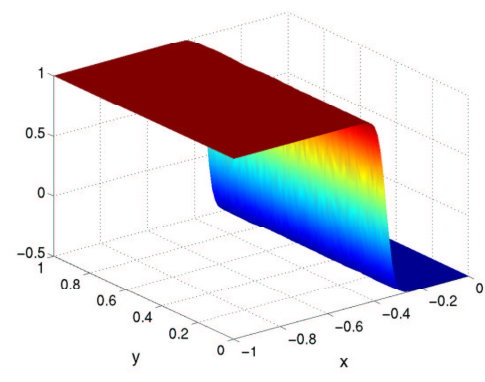

(a) $\epsilon_{1}=1$

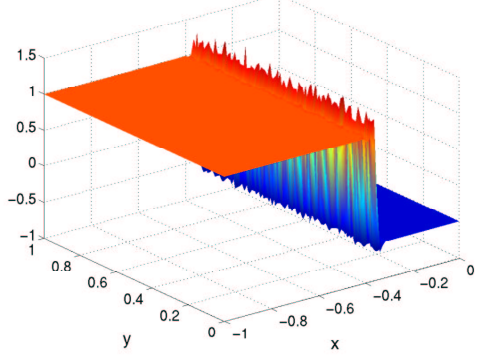

(b) $\epsilon_{4}=5$

Figure 5: Solutions at $t=2$ with different artificial dissipation. Test Case 1.

2. Initial data $f_{2}$, unstructured coarse mesh (2807 nodes) with either, $\epsilon_{1}=0, \epsilon_{4}=0$ or, $\epsilon_{1}=1, \epsilon_{4}=0$ or $\epsilon_{1}=0, \epsilon_{4}=5$. The shock will reach and pass through the boundary.

3. Initial data $f_{2}$, unstructured mesh with 11139 nodes with either, $\epsilon_{1}=$ $0, \epsilon_{4}=0$ or, $\epsilon_{1}=1, \epsilon_{4}=0$ or $\epsilon_{1}=0, \epsilon_{4}=5$. The shock will reach and pass through the boundary.

In Fig 5 Test Case 1 is shown. We see that the first-order dissipation yields a smooth shock. The fourth-order dissipation is not able to damp all oscillations at the shock but prevents them from spreading throughout the domain. In Fig 6, Test cases 2 and 3 are shown. On the coarse mesh all three options are stable. However, without dissipation the numerical solution has no accuracy and with dissipation the shock will move out through the boundary. The best performance is achieved with the first-order dissipation. On the fine mesh the non-dissipative even becomes unstable while the dissipative schemes manage to propagate the shock through the boundary.

Remark We have tuned the amount of dissipation to be efficient in this case only. To be efficient in general flow calculations it would need a limiter that identifies shocks and yield a proper scaling of the dissipation. Nonetheless, our computations shows that the structure of the artificial dissipation scheme is efficient.

\section{Conclusions}

We have constructed and analysed first-, second- and fourth-order Laplacian based dissipation operators and proven them to be stable and accurate. 


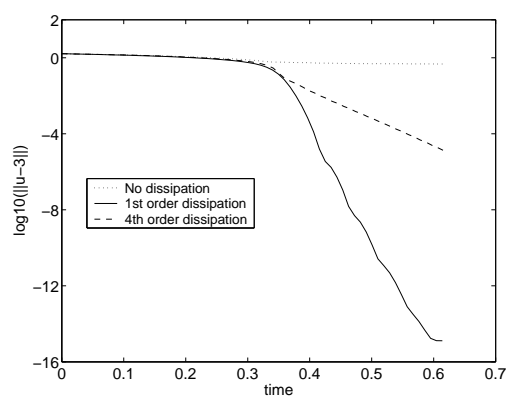

(a) Coarse mesh

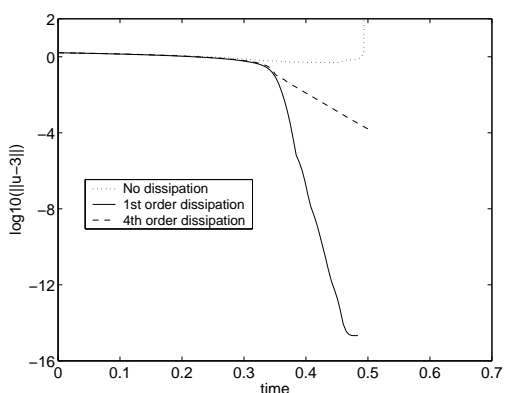

(b) Fine mesh

Figure 6: Deviation from steady state solution as a function of $t$. Test Cases 2 and 3.

The first-order dissipation is suitable for shocks and the fourth-order dissipation for non-physical oscillations. Computations corroborate the theoretical results.

\section{References}

[1] A. Haselbacher, J.J. McGuirk, and G.J. Page. Finite volume discretization aspects for viscous flows on mixed unstructured grids. AIAA Journal, 37(2), Feb. 1999.

[2] D.J Mavriplis. Accurate multigrid solution of the Euler equations on unstructured and adaptive meshes. AIAA Journal, 28(2), Feb. 1990.

[3] D.J Mavriplis. Multigrid strategies for viscous flow solvers on anisotropic unstructured meshes. J. Comput. Physics, 145, 1998.

[4] D.J. Mavriplis and D.W. Levy. Transonic drag prediction using an unstructured multigrid solver. Technical report, Institute for Computer Applications in Science and Engineering, 2002.

[5] D.J. Mavriplis and V. Venkatakrishnan. A unified multigrid solver for the Navier-Stokes equations on mixed element meshes. Technical report, Institute for Computer Applications in Science and Engineering, 1995.

[6] Jan Nordström, Karl Forsberg, Carl Adamsson, and Peter Eliasson. Finite volume methods, unstructured meshes and strict stability for hyperbolic problems. Applied Numerical Mathematics, 45(4), June 2003. 
[7] T. Gerhold, O. Friedrich, and J.Evans. Calculation of complex threedimensional configurations employing the $D L R$ - $\tau$-Code. In AIAA paper 97-0167, 1997.

[8] J.M. Weiss, J.P. Maruszewski, and W.A. Smith. Implicit solution of preconditioned Navier-Stokes equations using algebraic multigrid. AIAA Journal, 37(1), Jan. 1999.

[9] R.C. Swanson, R. Radespiel, and E.Turkel. On Some Numerical Dissipation Schemes. Technical Report 97-40, Institute for Computer Applications in Science and Engineering, 1997.

[10] Ken Mattsson, Magnus Svärd, and Jan Nordström. Stable and Accurate Artificial Dissipation. Journal of Scientific Computing, 21(1), August 2004.

[11] M. Svärd and J. Nordström. Stability of finite volume approximations for the laplacian operator on quadrilateral and triangular grids. Applied Numerical Mathematics, 51(1), October 2004.

[12] M. H. Carpenter, D. Gottlieb, and S. Abarbanel. Time-stable boundary conditions for finite-difference schemes solving hyperbolic systems: Methodology and application to high-order compact schemes. J. Comput. Phys., 111(2), 1994.

[13] M. H. Carpenter, J. Nordström, and D. Gottlieb. A Stable and Conservative Interface Treatment of Arbitrary Spatial Accuracy. J. Comput. Phys., 148, 1999.

[14] J. Nordström and M. H. Carpenter. Boundary and interface conditions for high-order finite-difference methods applied to the Euler and NavierStokes equations. J. Comput. Phys., 148, 1999.

[15] A. Haselbacher and J. Blazek. On the accurate and efficient discretisation of the Navier-Stokes equations on mixed grids. In AIAApaper 99-33552, 1999.

[16] D. Kuzmin, M. Möller, and S. Turek. High-resolution FEM-FCT schemes for multidimensional conservation laws. Technical Report 231, Ergebnisberichte Angewandte Mathematik, Universität Dortmund, 2003. 
The public reporting burden for this collection of information is estimated to average 1 hour per response, including the time for reviewing instructions, searching existing

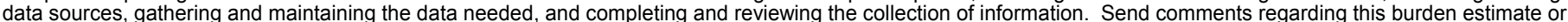

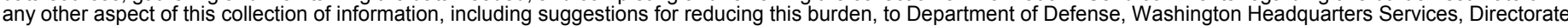

for Information Operations and Reports (0704-0188), 1215 Jefferson Davis Highway, Suite 1204, Arlington, VA 22202-4302. Respondents should be aware that

notwithstanding any other provision of law, no person shall be subject to any penalty for failing to comply with a collection of information if it does not display a currently

valid OMB control number.

PLEASE DO NOT RETURN YOUR FORM TO THE ABOVE ADDRESS

1. REPORT DATE (DD-MM-YYYY) 1 2. REPORT TYPE 3. DATES COVERED (FrOm - To)

01/04/2006

\section{TITLE AND SUBTITLE}

Contractor Report

Stable Artificial Dissipation Operators for Finite Volume Schemes on

Unstructured Grids 5a. CONTRACT NUMBER

5b. GRANT NUMBER

NCC1-02043

5c. PROGRAM ELEMENT NUMBER

5d. PROJECT NUMBER

2110

5e. TASK NUMBER

5f. WORK UNIT NUMBER

\section{PERFORMING ORGANIZATION NAME(S) AND ADDRESS(ES)}

NASA Langley Research Center

Hampton, VA 23681-2199

National Institute of Aerospace (NIA)

100 Exploration Way

Hampton, VA 23666
8. PERFORMING ORGANIZATION REPORT NUMBER

NIA Report No. 2005-05

10. SPONSORING/MONITOR'S ACRONYM(S)

NASA

11. SPONSORING/MONITORING REPORT NUMBER

NASA/CR-2006-214294

\section{DISTRIBUTION/AVAILABILITY STATEMENT}

Unclassified - Unlimited

Subject Category 64

Availability: NASA CASI (301) 621-0390

\section{SUPPLEMENTARY NOTES}

Langley Technical Monitor: Mark Carpenter

\section{ABSTRACT}

Our objective is to derive stable first-, second- and fourth-order artificial dissipation operators for node based finite volume schemes. Of particular interest are general unstructured grids where the strength of the finite volume method is fully utilised. A commonly used finite volume approximation of the Laplacian will be the basis in the construction of the artificial dissipation. Both a homogeneous dissipation acting in all directions with equal strength and a modification that allows different amount of dissipation in different directions are derived. Stability and accuracy of the new operators are proved and the theoretical results are supported by numerical computations.

\section{SUBJECT TERMS}

Finite Volume Method; Unstructured Grids; Artificial Dissipation; Stability; Accuracy

16. SECURITY CLASSIFICATION OF:

\begin{tabular}{|l|l|l|l|}
\hline a. REPORT & b. ABSTRACT & c. THIS PAGE & ABSTRACT \\
$\mathrm{U}$ & $\mathrm{U}$ & $\mathrm{U}$
\end{tabular}

\begin{tabular}{|l|l|}
\hline $\begin{array}{l}\text { 18. NUMBER } \\
\text { OF } \\
\text { PAGES }\end{array}$ & $\begin{array}{l}\text { 19b. NAME OF RESPONSIBLE PERSON } \\
\text { STI Help Desk (email: help@ sti.nasa.gov) }\end{array}$ \\
\cline { 2 - 3 } 23 & $\begin{array}{l}\text { 19b. TELEPHONE NUMBER (Include area code) } \\
\text { (301) } 621-0390\end{array}$ \\
\hline
\end{tabular}

